\title{
The role of self-esteem in the relationship between anxiety and depression of Albanian and Indian immigrants in Greece
}

\author{
Evangelia V. Kateri,, Argyroula Kalaitzaki, ${ }^{2}$ Evangelos C. Karademas ${ }^{1}$ \\ 'Department of Psychology, University of Crete, Rethymno, Crete \\ ${ }^{2}$ Social Work Department, Laboratory of Interdisciplinary Approaches for the Enhancement of Quality of Life, University Research Centre \\ "Institute of AgriFood and Life Sciences" Hellenic Mediterranean University, Heraklion, Crete, Greece
}

ARTICLE HISTORY: Received 14 September 2020/Revised 5 October 2020/Published Online 17 March 2021

\begin{abstract}
Immigrants' psychological health has been the focus of many studies as it is a timely subject due to the increasing numbers of immigrants and refugees who enter Greece the recent decades, and the resulting anxiety that this process brings about to the individual. The aim of the present study was to examine the relationship between immigrants' and Greeks' anxiety, self-esteem and depression. In addition, the present study aimed to compare the psychological health between immigrants and Greeks. The participants were 115 Albanian, 118 Indian immigrants, and 116 Greeks. The Rosenberg Self-Esteem Scale, the CES-D Scale, and the State Anxiety Inventory were administrated for measuring self-esteem, depression, and anxiety, respectively. To test the bivariate relationships between the study variables, Pearson product-moment correlation coefficients were calculated. The potential differences of psychological health between immigrant groups were examined with ANOVA, and multiple linear regression was used to predict the variance of depression by self-esteem and anxiety, after controlling for ethnicity and demographics. Moreover, moderation analysis was used to examine the moderation role of self-esteem in the relationship between anxiety and depression and possible differences between ethnic groups. In line with our hypotheses, immigrants had higher levels of depression and lower self-esteem scores, compared to Greeks. However, Indians reported the lower levels of anxiety compared to both Albanians and Greeks. Differences were also observed between the two immigrant groups, with Albanians experiencing more mental health problems than Indians. Both self-esteem and anxiety explained a large proportion of the variance of depression in immigrants (45\%), thus substantiating our theoretical model (i.e., depression depends on individuals' anxiety and self-esteem). Consistent to our expectations too, self-esteem was a moderator in the relationship between anxiety and depression; no differences between ethnic groups were observed though (e.g., the level of self-esteem acted protectively in the same way in Albanians, Indians, and Greeks). Despite the limitations, the findings of this study could be particularly useful to clinicians working with immigrants. Coping effectively with anxiety and enhancing immigrants' self-esteem could be tailored-based targets for both prevention and intervention programs.
\end{abstract}

KEYWORDS: Anxiety, self-esteem, depression, immigrants, Greece.

\section{Introduction}

During the recent decades, many immigrants, from both the Balkans and several Asian and African countries, have moved to Greece. Immigrants are regarded as a high-risk group for increased levels of depression and anxiety, as they are confronted with many stressors related to their adaptation to a new society. ${ }^{1,2}$ Major stressors are related to age, socioeconomic and educational status, years of residence in the host country, acculturation difficulties, and social exclusion., ${ }^{3,4}$ Cognitive theories assume that anxiety levels are high when a threat is perceived as potential, while depression levels are high when a threat is perceived as certain. Based on this assumption, anxiety and depression 
are hierarchically arranged, in that when the threat becomes imminent and certain the individual ends up in depression. ${ }^{5,6}$

Many studies indicate that the prevalence of depression and anxiety among immigrants is higher than the general population. ${ }^{7,8}$ Low educational level, old age, and few years of residence in the host country are regarded as risk factors for depression. ${ }^{7}$ Furthermore many studies examined the relationship between stressors, self-esteem, and health outcomes. Health outcomes vary between individuals and groups and self-esteem has a central role in this relationship- with both direct and indirect effects- and is regarded as a successful psychologically coping resource for stress. ${ }^{8}$ However, these findings are inconclusive as some studies have indicated that sociodemographic factors are unrelated to psychological health and immigrants not always report higher levels of anxiety and depression. ${ }^{8,9}$

Depression and anxiety have been associated with decreased self-esteem and impaired functioning in various domains of life. ${ }^{5}$ Terror management theory suggests that self-esteem protects individuals from anxiety that arises from the awareness of their vulnerability and mortality ${ }_{1}^{10}$ whereas Becks' cognitive theory of depression asserts that individuals with low self-esteem are more vulnerable to depression as they tend to focus on the negative aspects of the self, others, and the world. ${ }^{5}$ Previous studies suggest, also, that immigrants' self-esteem can protect them against anxious-depressed symptoms. ${ }^{11}$ However, self-esteem has also been linked with arrogance and narcissism, which are behaviors that many cultures discourage. ${ }^{12}$

Cultural differences in self-esteem have been found due to differences in the construction of the self. In more individualistic countries (e.g. usually western countries), individuals are primarily characterized by independent self-construal, perceiving themselves to be separate from others and autonomous, while in more collectivistic countries (e.g. usually non-western countries) individuals are primarily characterized by interdependent self-construal, perceiving themselves to be defined by their relations to others. ${ }^{13-15}$ Several cross-cultural studies have shown that in individualistic cultures, people engage in self-enhancement strategies, perceiving their superiority over the others and aiming at accomplishing personal goals ${ }^{8}$ whereas in collectivistic countries, the self is interrelated to significant others and people are motivated to perceive themselves as inferior to others, aiming at being accepted by the in-group. ${ }^{13,16}$ Therefore, lower self-esteem rates for people coming from collectivistic countries compared to people coming from individualistic countries have been found in many studies. ${ }^{16}$ Furthermore, self-esteem ameliorates the negative effects of immigrants' stress $s^{17,18}$ by interpreting daily stressors more positively, seeking for social support, and trying harder to adapt to the host country. ${ }^{19,20}$ Indian self is characterized mainly by values of interdependence, ${ }^{21-23}$ while the Greek self is characterized by both interdependence and independence ${ }^{24}$ or by both individualistic and collectivistic values ${ }^{25}$ and these values affect self-esteem. ${ }^{13}$

Based on the controversial evidence on the relationship between immigration, depression, anxiety and self-esteem, $, 8,9,12,16$ the present study aimed to examine whether the high levels of anxiety result in depression and whether the relationship between anxiety and depression is moderated by self-esteem in two groups of immigrants (Albanians and Indians) and compare them with a Greek sample of similar sociodemographic characteristics (see figure 1). To the authors' knowledge no other studies have examined the interrelationship of these variables in three ethnicity groups, immigrants and hosts.

Given that immigration is a stressful condition, ${ }^{1,2,7}$ it was hypothesized that immigrants (both Albanian and Indians) would present higher levels of anxiety and depression compared to Greeks. Longer residence in Greece, higher education level, and younger age, were expected to correlate negatively with anxiety and depression. ${ }^{3,4,7}$

Self-esteem was expected to be a moderator in the relationship between anxiety and depression. ${ }^{10}$ Significant differences in self-esteem were expected between the three ethnic groups (e.g., Albanians, Indians, Greeks). Given that self-esteem rates are lower for people coming from collectivistic countries, $13,16,23$ Indians were expected to have low levels of self-esteem, Greeks high levels ${ }^{24}$ and Albanians moderate levels. ${ }^{25,26}$ Although no previous study has examined cultural values in Albanians, they are considered to be culturally closer to Greeks than immigrants coming from Asian and African countries, ${ }^{27}$ suggesting also that their self-esteem level would probably lie somewhere between Greeks and Indians.

Since Albanians have been integrated or/and assimilated to Greek society, ${ }^{2,27}$ while Indians are more separated $^{22}$ and integrated or/and assimilated immigrants usually report higher levels of self-esteem than sep-

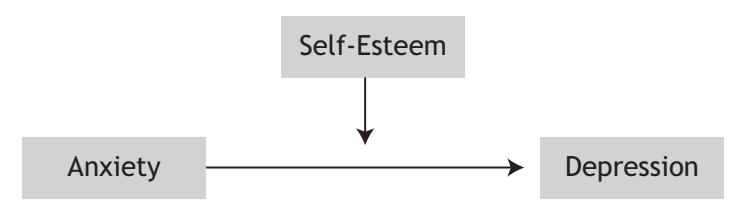

Figure 1. The theoretical model of the present study. 
arated ones, ${ }^{12,28}$ it was expected that, the higher levels of self-esteem would act protectively for Greeks, the medium levels for Albanians and the lower levels for Indians. Possible differences in anxiety and depression of Albanians and Indians were also examined, given that culture affect mental health. ${ }^{13}$ It was expected that Indians will present less anxiety and depression symptoms compared to Albanians, because of Indians interdependence ${ }^{23}$ and their commitment to their heritage culture group that increase in-group social support. ${ }^{29}$

\section{Material and Method}

\section{Participants}

Immigrants. Two hundred and thirty-three immigrants living in Greece ( $M=33.0, S D=8.0$ years old) participated in the study. One hundred and fifteen five of them were Indians and one hundred and eighteen were Albanians. Their average age was 33.0 years for Indians $(S D=8.0)$ and 35.0 years for Albanians $(S D=8.0)$. Their average years of education were 11.2 years $(S D=2.7)$ for Indians and 11.8 years $(S D=2.4)$ for Albanians. Indians and Albanians were residing in Greece $7.7(S D=3.9)$ and 10.7 $(\mathrm{SD}=4.2)$ years, respectively.

Greeks. One hundred and sixteen were Greeks. Their average age was $39.0(\mathrm{SD}=9)$ and their average years of education were $12.0(\mathrm{SD}=2.8)$.

\section{Measures}

A number of demographic information, including gender, age, educational level, ethnicity, years of residence in Greece (only for immigrants) were collected. Cronbach's alpha coefficients for all scales are presented in table 1.

Self-Esteem. Self-esteem was measured with the SelfEsteem Scale. ${ }^{30}$ It consists of 10 items that assess the global personal self-esteem (e.g., "Sometimes I feel totally usefulness"). Participants respond on a Likerttype scale ranging from "totally disagree" (1) to "totally agree" (7).
Depression symptoms. Depression was measured with the Center for Epidemiological Studies-Depression scale (CES-D). ${ }^{31}$ It consists of 20 items (e.g., "I felt hopeful about the future") that cover affective, psychological, and somatic symptoms of depression. Participants respond on a Likert-type scale ranging from "strongly disagree" (1) to "strongly agree" (5).

Anxiety symptoms. Anxiety was measured with the State Anxiety Inventory ${ }^{32}$ which evaluates the anxiety the person feels at the moment of measurement. It consists of 20 items (e.g., "I feel calm") and participants respond on a Likert-type scale ranging from "not at all" (1) to "very much" (4).

\section{Procedure and analyses}

Participants were recruited at their workplace. Indians were also recruited in a building that they use for their religious ceremonies. A convenience sampling technique was used. Participation in the study was voluntary. Each measure was translated from English to Punjab and Albanian and back-translated to English. ${ }^{33}$ All analyses were carried out with SPSS 24.0 and the moderation analysis with PROCESS, which is a freely available computational tool for SPSS and SAS. ${ }^{34}$ The moderation effects were considered significant at $p<0.05$ for the $95 \%$ bootstrap confidence intervals when the derived intervals do not include values of zero. ${ }^{34}$

\section{Results}

Albanians had lived statistical significantly (t (219)= $-5.38, p<0.001)$ more years in Greece $(M=10.7, S D=4.2)$ compared to Indians ( $M=7.7, S D=3.9)$. Years of education did not differentiate the three ethnic groups (Albanians, Indians, and Greeks) $(F 2,325=2.39, \mathrm{p}>0.05)$. The difference between Indians' mean age $(M=33, S D=8)$ and Albanians' $(M=35, S D=8)$ was not statistically significant (t $(223)=-1,23, p>0.05)$, but it was between immigrants and Greeks $(M=39, S D=9)(F 2,337=14.12, p<0.001)$.

Table 1. Bivariate Correlations among immigrants' anxiety, depression, self-esteem, age, education, and years of tesidence.

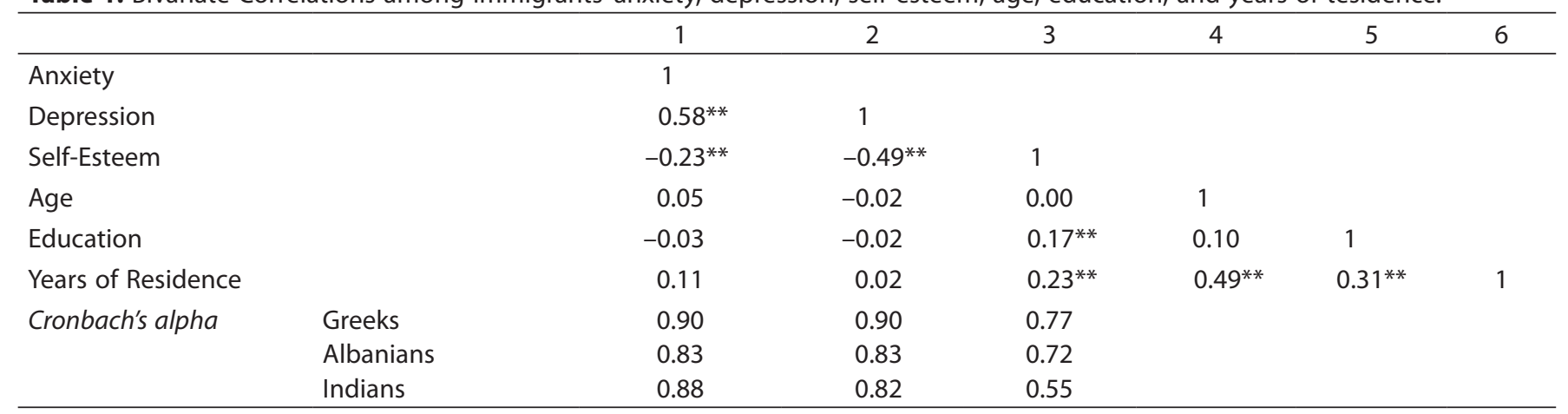

${ }^{*} p<0.05 ;{ }^{* *} p<0.01$ 
One-way ANOVAs indicated statistically significant differences between the three ethnic groups (i.e., Albanians, Indians, and Greeks) regarding anxiety ( $F 2,249=5.611$, $p<0.01)$, depression $(F 2,297=4.59, p<0.05)$, and self-esteem $(F 2,314=20.27, p<0.00)$. Albanians had higher levels of anxiety $(M=47.16, S D=10.70)$, followed by Greeks $(M=44.30, S D=11.88)$ and Indians $(M=41.52, S D=8.27)$. Albanians had, also, higher levels of depression symptoms $(M=20.42, S D=9.75)$, followed by Indians $(M=17.42$, $S D=9.64)$ and Greeks $(M=16.12, S D=10.75)$. Greeks had higher self-esteem scores $(M=54.81, S D=7.93)$, followed by Albanians $(M=52.45, S D=8.67)$ and Indians $(M=47.0$, $\mathrm{SD}=7.72)$.

Table 1 presents the bivariate correlations among immigrants' age, education, years of residence in Greece, self-esteem, anxiety, and depression. Education was statistically significant positively correlated with self-esteem $(r=0.17, p<0.005)$. Anxiety was statistically significant correlated positively with depression $(r=0.58$, $p<0.005)$ and negatively with self-esteem $(r=-0.23$, $p<0.005)$, and self-esteem was negatively correlated with both anxiety and depression $(r=-0.23, p<0.005$ and $r=-0.49, p<0.005$, respectively).

Subsequently, a hierarchical multiple regression analysis was carried out, aiming to examine the degree that ethnicity, age, education, and years of residence (step1), and self-esteem and anxiety (step 2) predict depression symptoms in immigrants. Step 1 predicted $0.04 \%$ of variance, $F(4,112)=2.19, p>0.05$. According to Beta values, only ethnicity was a significant predictor of depression. When self-esteem and anxiety were added, the variance of depression changed $0.40 \%(F(2,110)=42.72, p<0.00)$. Both variables predicted $45 \%$ of the variance of the outcome variable (see table 2 ).

Model 1 of PROCESS was then performed to test whether self-esteem moderates the relationship between anxiety and depression, separately for Greeks, Albanians, and Indians. As shown in table 3, the correlation between anxiety and depression was statistical- ly significant at the higher (+1 SD), at the medium (M), and at the lower levels of self-esteem ( $-1 \mathrm{SD})$ for all ethnic groups. However, for Indians, the high level of self-esteem was less significant than that of Greeks and Albanians $(\mathrm{B}=0.44, \mathrm{SE}=0.20, \mathrm{p}<0.05, \mathrm{Cl}=0.03$ to 0.86 , $\mathrm{B}=0.54, \mathrm{SE}=0.11, \mathrm{p}<0.01, \mathrm{Cl}=0.32$ to 0.76 , and $\mathrm{B}=0.33$, $\mathrm{SE}=0.08, \mathrm{p}<0.01, \mathrm{Cl}=0.16$ to 0.50 , respectively).

\section{Discussion}

In line with our hypothesis, both Albanian and Indian immigrants, compared to Greeks, reported higher rates of depression and lower rates of self-esteem. Interestingly, Albanians also reported higher rates of anxiety compared to Greeks, but Indians reported lower levels of anxiety from Greeks, contrary to our expectations. These results suggest that immigration could be a risk factor for health decline. ${ }^{3,4}$ However, cultural differences in anxiety have been reported worldwide ${ }^{35}$ as well as coping mechanisms (that were not examined in this study). Indians obtain meaning in life through their belief in Gods or other spirits, and religion is a typical and effective coping mechanism to deal with anxiety. ${ }^{36,23}$ Albanians reported higher anxiety and depression compared to Indians, supporting our hypothesis. Immigrants in Greece experience discrimination and are generally perceived as a threat to Greek cultural identity, social security and welfare systems. ${ }^{37}$ However, when immigrants emotionally invest in their ethnic group and maintain their original cultural traits, they seem to be more protected from discrimination than the immigrant groups who strive to be incorporated to the host society. ${ }^{29,38}$ Previous findings indicate that Indians' separation from hosts was related to more social support, ending, in turn, to fewer depressive symptoms. ${ }^{22}$

Contrary to our expectations, demographics, such as years of residence in Greece, education, and age did not correlate nor were they predictors of anxiety and depression. A possible explanation is that immigrant's mental health might be more complicated than expect-

Table 2. Hierarchical regression analysis for the prediction of depression in Albanian and Indian immigrants.

\begin{tabular}{|c|c|c|c|c|c|c|}
\hline & Beta & $\mathrm{t}$ & Adj. $\mathrm{R}^{2}$ & $\mathrm{R}^{2}$ Change & F Change & DF \\
\hline Step 1 & & & 0.04 & 0.07 & 2.19 & 4,112 \\
\hline Ethnicity & 0.27 & $0.27^{* *}$ & & & & \\
\hline Age & -0.08 & -0.81 & & & & \\
\hline Education & -0.05 & -0.05 & & & & \\
\hline Years of residence & -0.04 & -0.35 & & & & \\
\hline Step 2 & & & 0.45 & 0.40 & $42.72^{* * *}$ & 2,110 \\
\hline Self-Esteem & -0.41 & $-5.32^{* * *}$ & & & & \\
\hline Anxiety & 0.39 & $4.8^{* * *}$ & & & & \\
\hline
\end{tabular}

${ }^{* *} p<0.01 ;{ }^{* * *} p<0.001$ 
Table 3. Bootstrapping Results for moderation effects of self-esteem on the relationship of anxiety to depression for Greeks, Indians, and Albanians.

\begin{tabular}{|c|c|c|c|c|c|c|}
\hline \multirow[b]{2}{*}{ Self-esteem } & \multirow[t]{2}{*}{ B } & \multirow[t]{2}{*}{ SE } & \multirow[t]{2}{*}{$\mathrm{t}$} & \multirow[t]{2}{*}{$p$} & \multicolumn{2}{|c|}{$95 \% \mathrm{Cl}^{\dagger}$} \\
\hline & & & & & Lower & Upper \\
\hline \multicolumn{7}{|l|}{ Greeks } \\
\hline Mean & $0.53^{* * *}$ & $0.09 * * *$ & 5.39 & $<0.01$ & 0.33 & 0.73 \\
\hline High (+1SD) & $0.54^{* * *}$ & $0.11^{* * *}$ & 4.95 & $<0.01$ & 0.32 & 0.76 \\
\hline Low (-1SD) & $0.59^{* * *}$ & $0.18^{* * *}$ & 3.14 & $<0.01$ & 0.21 & 0.97 \\
\hline Mean & $0.52^{* * *}$ & $0.14^{* * *}$ & 3.65 & $<0.01$ & 0.23 & 0.80 \\
\hline High (+1SD) & $0.44^{*}$ & $0.20^{*}$ & 2.18 & 0.03 & 0.03 & 0.86 \\
\hline \multicolumn{7}{|l|}{ Albanians } \\
\hline Low (-1SD) & $0.37^{* * *}$ & $0.13^{* * *}$ & 2.87 & $<0.01$ & 0.11 & 0.64 \\
\hline
\end{tabular}

${ }^{\dagger}$ Bootstrapping bias corrected and accelerated (5000 bootstrap samples). Intervals that do not contain zero are deemed to be significant. Note: $\mathrm{SD}=$ standard deviation. $\mathrm{Cl}=$ confidence intervals, ${ }^{*} \mathrm{p}<0.05,{ }^{* *} \mathrm{p}<0.001$.

ed; many factors other than demographics may affect their mental health or act as mediators or moderators in this relationship. ${ }^{39}$ Education, for example, may offer more resources (cognitive, economic, and social) to deal with changes, ${ }^{26}$ while longer residence in the host country possibly extents social networks ${ }^{7,40}$ which boost their self-esteem. Future studies need to test these assumptions.

As expected, immigrants' self-esteem and anxiety explained a high proportion of the variance of depression, suggesting that the relationship of anxiety, self-esteem and depression ${ }^{5,6,10}$ could be etic or universal. In line with previous studies, ${ }^{16}$ Indians reported the lowest rates of self-esteem, Greeks the highest ones, and Albanians moderate rates, supporting our hypothesis. This finding is indicative of the cultural differences (independence/interdependence) related to self-esteem. The higher the independent values, the higher the self-esteem. ${ }^{26}$ The results also provided support to the moderating role of self-esteem in the relationship between anxiety and depression in the three sampling groups, indicating that self-esteem protects individuals from depression, ${ }^{17,11}$ no matter if the individual is an immigrant or host. Previous studies found small differences in the protective role of self-esteem between immigrants and no immigrants as well. ${ }^{28}$

Contrary to our expectations, no differences in self-esteem were found between ethnic groups in the levels of self-esteem that act protectively. It was expected, for example, Indians low self-esteem would act protectively, compatible with their collectivistic values of modesty and inferiority. ${ }^{13}$ The only indication in line with this hypothesis was that Indians high self-esteem was less statistically significant than that of Greeks and Albanians. A crucial question is if these results represent incompetency of the measures. Self-esteem can be measured as an overall index or comprising of two factors: personal abilities that are reported more in individualistic cultures and the desirability of the individuals' behavior to significant others that is reported more in collectivistic cultures. ${ }^{41}$ Indians' self-esteem may be more related to social worth and feelings of interrelatedness with significant others or collective self-esteem ${ }^{42}$ than to intrapersonal feelings and intrapersonal evaluations of success (i.e. abilities), that the Rosenberg Self-Esteem Scale measures. ${ }^{40,43}$ Cultural values of independence and interdependence must be measured in future studies and not ascribed a priori to ethnicity ${ }^{44}$ The measurement of collective self-esteem is also suggested in future studies.

The limitations of this study should be acknowledged. The most important limitations are the cross-sectional nature of the study ${ }^{45}$ and the use of a small convenient sample, which limit the generalizability of the findings. The reliability of the self-esteem scale was also low for Indians, raising concerns about the cross-cultural validity of this scale in non-Western cultures. ${ }^{46} \mathrm{~A}$ prospective study should be carried out in many immigrant groups and in both clinical and non-clinical samples.

Despite the limitations, the implications of the present study are noteworthy. The present findings make a unique theoretical contribution to the protective 
role of self-esteem in immigrants and hosts. Although self-esteem is a protective factor for depression, the Indians lower self-esteem levels, compared to Greeks and Albanians, might be more compatible with their cultural values. Clinicians must be cautious with generalizations and interpret behavior in line with the clients' cultural context. Indisputably, immigrants are one of the most vulnerable population subgroups that face significant difficulties and stressors. Being already

\section{References}

1. Berry JW, Sabatier C. Variations in the assessment of acculturation attitudes: Their relationships with psychological wellbeing. Int $\mathrm{J}$ Intercult Rel 2011, 35:658-669, doi: 10.1016/j.ijintrel.2011.02.002

2. Besevegis E, Pavlopoulos V. Acculturation patterns and adaptation of immigrants in Greece. In: Finklestein M, Dent- Brown K (eds) Psychosocial stress in immigrants and in members of minority groups as a factor of terrorist behaviour. IOS Press, 2008, doi: 10. 3233/9781-58603-872-4-23

3. Madianos M. Immigration and repatriation: A continuum effect of mental disorders. In: Papastylianou A (ed) Cross-Cultural Routes. Ellinika Grammata, Athens, 2005 (In Greek)

4. Mpilanakis N. Immigration and repatriation: A continuum effect of mental disorders. In: Papastylianou A (ed) Cross-Cultural Routes. Ellinika Grammata, Athens, 2005 (In Greek)

5. Sowislo JF, Orth U. Does low self-esteem predict depression and anxiety? A meta-analysis of longitudinal studies. Psychol Bull 2013, 139: 213-240, doi: 10.1037/a0028931

6. Dobson KS. The relationship between anxiety and depression. Clin Psychol Rev 1985, 5:307-324, doi: 10.1016/0272-7358(85)90010-8

7. Berry JW. Immigration, acculturation and adaptation. Appl Psychol-Int Rev 1997, 46:5-34, doi: 10.1111/j.1464-0597.1997.tb01087.x

8. Noh S, Avison WR. Asian Immigrants and the Stress Process: A Study of Koreans in Canada. J Health Soc Beh 1996, 37:192-206, doi: $10.2307 / 2137273$

9. Koneru VK, Weisman de Mamani AG, Flynn PM, Betancourt H. Acculturation and mental health: Current findings and recommendations for future research. Appl Prev Psychol 2007, 12:76-96, doi: 10.1016/j.appsy.2007.07.016

10. Greenberg J, Simon L, Solomon S, Chatel D, Pyszczynski T. Terror Management and Tolerance: Does Mortality Salience Always Intensify Negative Reactions to Others Who Threaten One's Worldview. J Pers Soc Psychol 1992, 63:212-220, doi: 0022-3514/92/S3.00

11. Okazaki S, Ryce P, Sirin SR. The Role of Collective Self-Esteem on Anxious-Depressed Symptoms for Asian and Latino Children of Immigrants. Cultural Diversity and Ethnic Minority Psychology 2014, 20:220-230, doi: 10.1037/a0035022

12. Konrath S. Self-esteem, culturally defined. In: Scull A (ed) Cultural Sociology of Mental Illness: An A-to-Z Guide. Sage, 2014, doi: 10.4135/9781483346342

13. Markus HR, Kitayama S. Culture and the self: Implications for cognition, emotion and motivation. Psychol Rev 1991, 98:224-253, doi: 10.1037/0033-295X.98.2.224

14. Singelis TM. The measurement of independent and interdependent self-construals. Pers Soc Psychol Bull 1994, 20:580-591, doi: 10.1177/ 0146167294205014

15. Smith PB, Bond MH. Social psychology across cultures. Prentice Hall, London, 1998 severely stressed and experiencing also the COVID-19 pandemic may be very risky for their psychological health. Although many of the stressors they face cannot be prevented, these populations need urgently to be safeguarded from the negative consequences they experience in the host country. Clinicians and other stakeholders can target modifiable factors such as enhancing their self-esteem with long-term benefits in minimizing the risk of depression. ${ }^{17}$

16. Tsai JL, Ying YW, Lee PA. Cultural predictors of self-esteem: A study of Chinese American female and male young adults. Cult Divers Ethn Min Psychol 2001, 7:284-297, doi: 10.1037/1099-9809.7.3.284

17. Hovey J, Magana S. Acculturative Stress, Anxiety, and Depression among Mexican Immigrant Farmworkers in the Midwest United States. J Immigr Health 2000, 2:119-131, doi: 1096-4045/00/0700-011918.00/0

18. Nesdale D, Mak AS. Ethnic identification, self-esteem and immigrant psychological health. Int J Intercult Rels 2003, 27:23-40, doi: 10.1016/ S0147-1767(02)00062-7

19. Cross SE, Gore JS, Morris ML. The relational-interdependent self-construal, self-concept consistency, and well-being. J Pers Soc Psychol 2003, 85:933-944, doi: 10.1037/0022-3514.85.5.933

20. Kosic A. Personality and individual factors in acculturation. In: Sam D, Berry JW (eds) The Cambridge Handbook of Acculturation Psychology. University Press, Cambridge, 2006

21. Kapoor S, Hughes PC, Baldwin JR, Blue J. The relationship of individualism-collectivism and self-construals to communication styles in India and the United States. J Intercult Relat 2003, 27:683-700, doi: 10.1016/j.jijintrel.2003.08.002

22. Kateri E, Tzouvelas G, Karademas E. The role of acculturation attitudes and social support in anxiety and depression of Indian immigrants in Greece. Psychiatriki 2019, 30:311-319, doi: 10.22365/ jpsych.2019.304.311

23. Kateri E, Karademas E. The interplay between self-construal, social support, and psychological adaptation of Indian immigrants' in Greece. Eur J Couns Psychol 2018, 7:148-164, doi: 10.5964/ejcop.v7i1.148

24. Green EG, Deschamps JC, Paez D. Variation of individualism and collectivism within and between 20 countries: A typological analysis. $J$ Cross Cult Psychol 2005, 36:321-339, doi: 10.1177/0022022104273654

25. Kafetsios K, Nezlek JB. Attachment and wellbeing: A study of emotion and social support in everyday interaction. Paper presented at the Biannual Conference of the International Association for Relationship Research, University of Madison Wisconsin, Madison, 2004

26. Diener E, Diener M. Cross-Cultural Correlates of Life Satisfaction and Self-Esteem. Pers Processes Individ Dif 1995, 68:653-666, doi: 10.1037//0022-3514.68.4.653

27. Tsouvelas G, Pavlopoulos V. Greek host community acculturation expectations towards immigrants from Albania and Pakistan: The role of existential parameters. Eur J Couns Psychol 2018, 7:181-199, doi: 10.5964/ejcop.v7i1.151

28. Portes PR, Zady MF. Self-Esteem in the Adaptation of Spanish-Speaking Adolescents: The Role of Immigration, Family Conflict, and Depression. Hispanic J Behav Sci 2002, 24:296-318, doi: 10.1177/0739986302024003003

29. Gungor D. The interplay between values, acculturation and adaptation: A study on Turkish- Belgian adolescents. Int J Psych 2007, 42:380-392, doi: 10.1080/00207590600878657

30. Rosenberg M. Society and the adolescent self-image. Princeton University Press, Princeton, 1965 
31. Radloff LS. The CES-D scale: A self-report depression scale for research in the general population. Appl Psych Meas 1977, 1:385-401, doi: $10.1177 / 014662167700100306$

32. Spielberger GD, Gorush RL, Lushene RE. The State-Trait Anxiety Inventory. CA: Consulting Psychologists Press, Palo Alto, 1970

33. Brislin RW. Back-translation for cross-cultural research. J Cross Cult Psychol 1970, 1:185-216, doi: 10.1177/135910457000100301

34. Hayes E. An introduction to mediation, moderation, and conditional process analysis: A regression-based approach. Guilford Press, New York, 2013

35. Shiraev EB, Levy DA. Cross-Cultural Psychology: Critical Thinking and Contemporary Applications. Sixth ed. Routledge, New York and London, 2017

36. Kateri E, Pourkos M. Epistemological considerations about the psychoeducational and psychotherapeutic approach of the cultural other. In: Pourkos M (ed) Socio-Historical-Cultural Approaches in Psychology and Education. Atrapos Publications, Athens, 2006 (In Greek)

37. Triantafyllidou A. The political discourse on immigration in southern Europe: A critical analysis. J Community App/ Soc Psychol 2000, 10:373-389, doi: 10.1002/1099-1298

38. Jasinskaja-Lahti I, Liebkind K, Horenczyk G, Schmitz P. The interactive nature of acculturation: perceived discrimination, acculturation attitudes and stress among young ethnic repatriates in Finland, Israel and Germany. Int J Intercult Rel 2003, 27:79-97, doi: 10.1016/S01471767(02)000061-5
39. Salant T, Lauderdale DS. Measuring Culture: A critical review of acculturation and health in Asian immigrant populations. Soc Sci Med 2003, 35:1-20, doi: 10.1016/S0277-9536(02)00300-3

40. Jasinskaja-Lahti I, Liebkind K, Jaakkola M, Reuter A. Perceived discrimination, social support networks, and psychological well-being in three immigrant groups. J Cross Cult Psychol 2006, 37:293-311, doi: 10.1177/0022022106286925

41. Tafarodi RW, Lang JM, Smith AJ. Self-esteem and the cultural trade-off: Evidence for the role of individualism collectivism. J Cross Cult Psychol 1999, 30:620-640, doi: 10.1177/0022022199030005

42. Branscombe NR, Wann DL. Collective self-esteem consequences of outgroup derogation when a valued social identity is on trial. Eur J Soc Psychol 1994, 24:641-657, doi: 10.1002/ejsp.2420240603

43. Mesquita B. Emotions in collectivist and individualist contexts. $J$ Pers Soc Psychol 2001, 80:68-74, doi:10.1037/0022-3514.80.1.68

44. Triandis HC. Culture and social behavior. McGraw- Hill, Inc, New York, 1994

45. Maxwell SE, Cole DA. Bias in cross-sectional analyses of longitudinal mediation. Psychol Methods 2007, 12: 23-44, doi: 10.1037/1082989X.12.1.2

46. Oyserman $D$, Lee SW. Does culture influence what and how we think? Effects of priming individualism and collectivism. Psychol Bull 2008, 134: 311-342, doi: 10.1037/0033-2909.13 


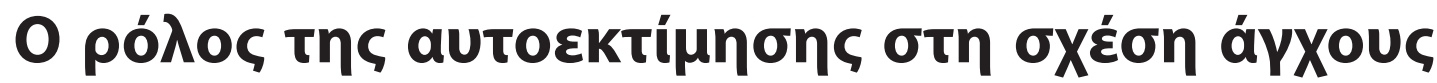

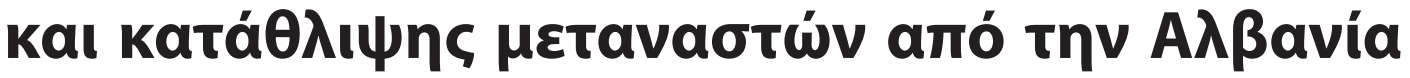

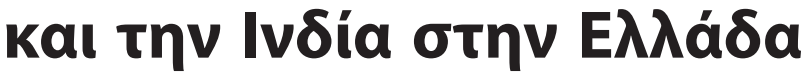

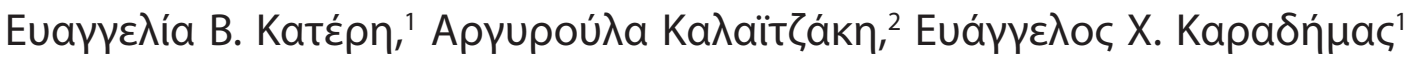

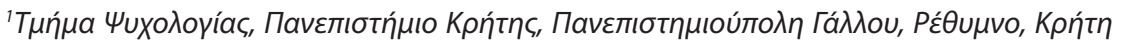

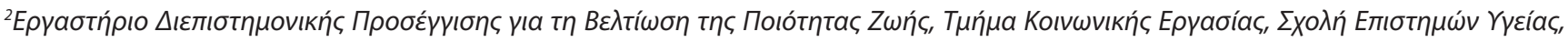

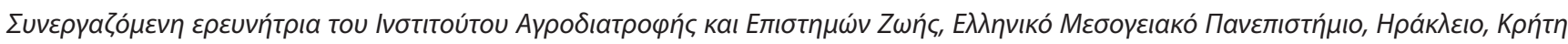

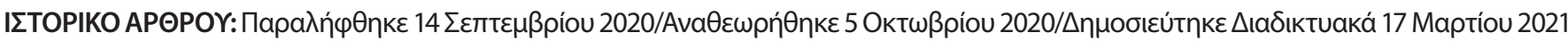

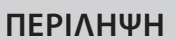

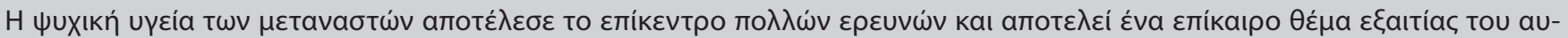

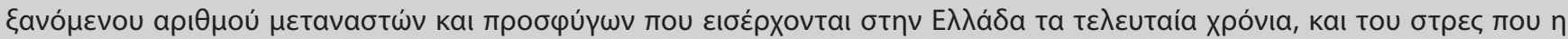

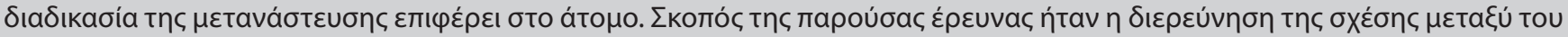

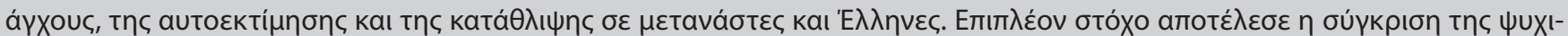

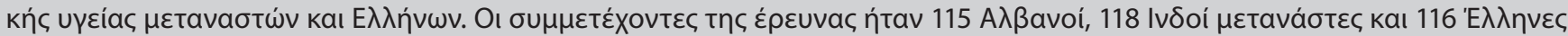

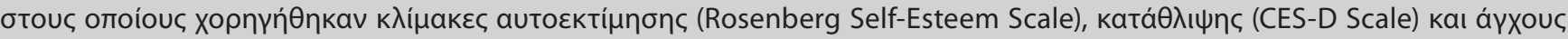

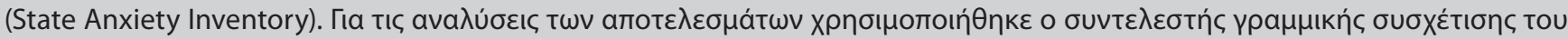

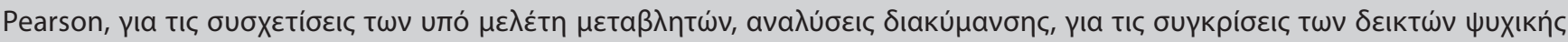

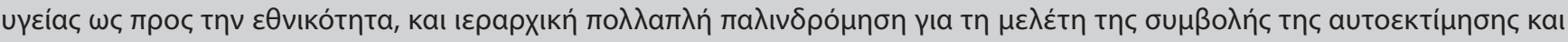

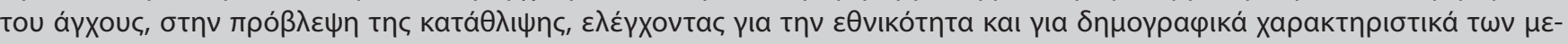

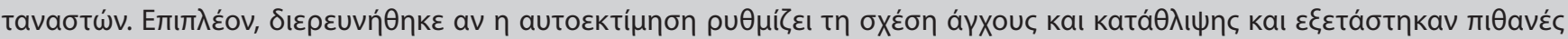

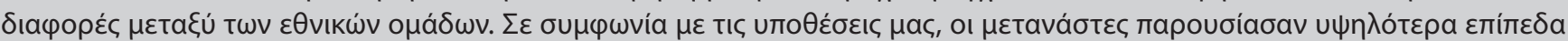

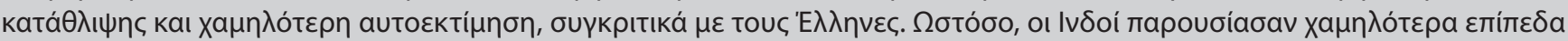

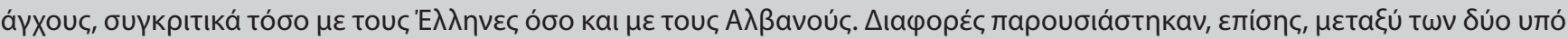

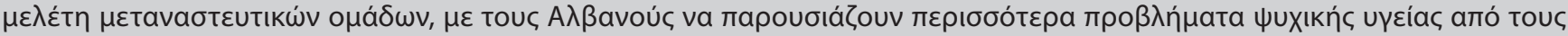

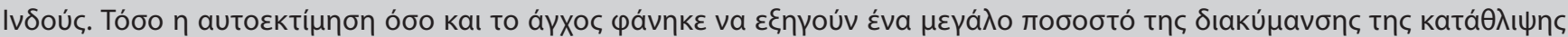

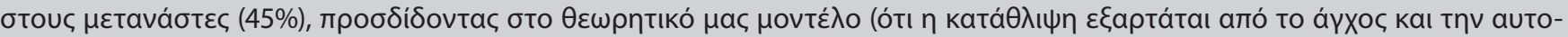

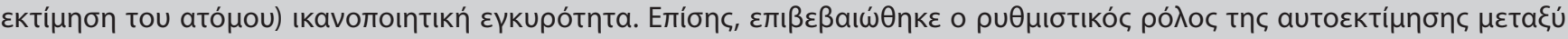

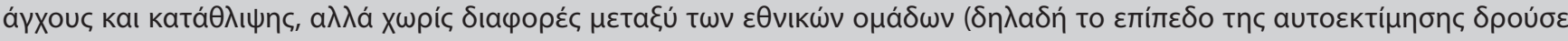

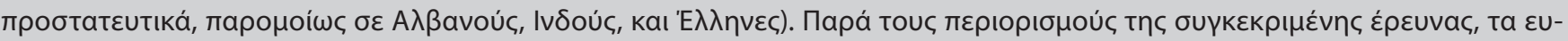

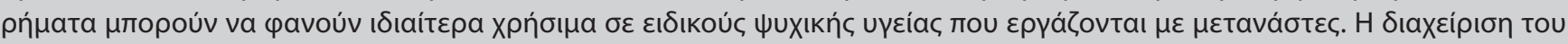

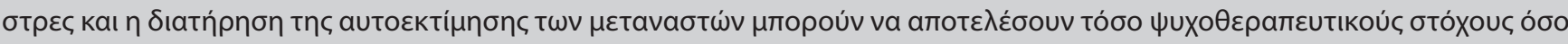

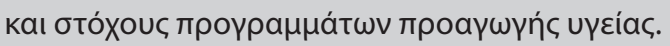

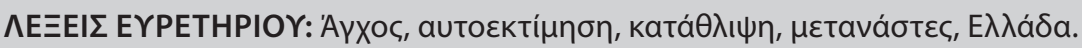

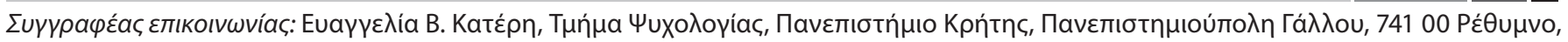

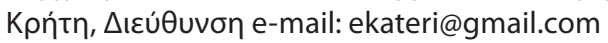

